\title{
Stochastic model of CO oxidation on platinum surfaces and deterministic limit
}

Cite as: J. Chem. Phys. 115, 4829 (2001); https://doi.org/10.1063/1.1391255

Submitted: 07 March 2001 . Accepted: 19 June 2001 . Published Online: 24 August 2001

Christian Reichert, Jens Starke, and Markus Eiswirth

\section{ARTICLES YOU MAY BE INTERESTED IN}

Oscillatory CO oxidation on Pt(110): Modeling of temporal self-organization

The Journal of Chemical Physics 96, 9161 (1992); https://doi.org/10.1063/1.462226

Parallel kinetic Monte Carlo simulation framework incorporating accurate models of adsorbate lateral interactions

The Journal of Chemical Physics 139, 224706 (2013); https://doi.org/10.1063/1.4840395

Molecular beam study of CO oxidation on a (111) platinum surface

The Journal of Chemical Physics 60, 1453 (1974); https://doi.org/10.1063/1.1681219

\section{Lock-in Amplifiers up to $600 \mathrm{MHz}$}
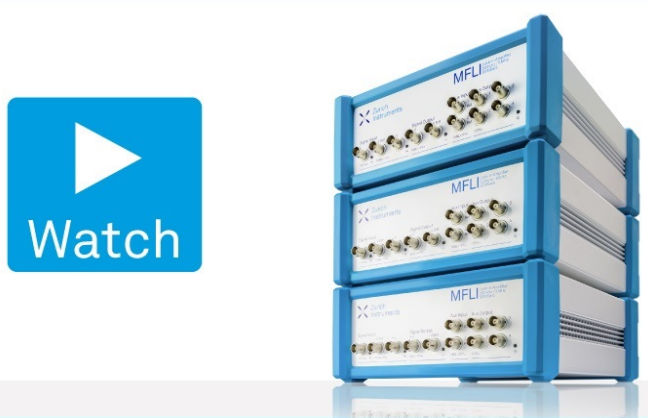

J. Chem. Phys. 115, 4829 (2001); https://doi.org/10.1063/1.1391255 


\title{
Stochastic model of $\mathrm{CO}$ oxidation on platinum surfaces and deterministic limit
}

\author{
Christian Reichert \\ Interdisciplinary Center for Scientific Computing, University of Heidelberg, Im Neuenheimer Feld 294, \\ D-69120 Heidelberg, Germany \\ Jens Starke ${ }^{\text {a) }}$ \\ Interdisciplinary Center for Scientific Computing \& Institute of Applied Mathematics, \\ University of Heidelberg, Im Neuenheimer Feld 294, D-69120 Heidelberg, Germany \\ Markus Eiswirth \\ Fritz Haber Institute of the Max Planck Society, Faradayweg 4-6, D-14195 Berlin, Germany
}

(Received 7 March 2001; accepted 19 June 2001)

\begin{abstract}
We present a general model for the oxidation of CO on low-index platinum single crystal surfaces. In order to take fluctuations into account, it is first formulated in terms of stochastic birth-death processes. A corresponding deterministic limit for large particle numbers can be derived rigorously. The dynamical behavior of the reaction kinetics is investigated by means of a numerical bifurcation analysis of the deterministic limit and stochastic simulations. The computed bifurcation diagrams for $\mathrm{Pt}(110)$ and $\mathrm{Pt}(100)$ are in qualitative and reasonable quantitative agreement with experimental results. For $\mathrm{Pt}(110)$, several types of noise-induced oscillations are obtained. (C) 2001 American Institute of Physics. [DOI: 10.1063/1.1391255]
\end{abstract}

\section{INTRODUCTION}

The temporal and spatiotemporal dynamics of heterogeneously catalyzed reactions has been investigated for a few decades. ${ }^{1-3}$ Experimental studies on well-defined single crystal surfaces using surface science techniques allowed to elucidate the mechanisms of a number of surface reactions by clarification of the underlying elementary steps and direct measurement of relevant kinetic parameters.

A system that has been studied extensively is the CO oxidation on low-index plane platinum surfaces which exhibits a large variety of spatiotemporal patterns. ${ }^{4}$ This reaction was found to follow a Langmuir-Hinshelwood mechanism with asymmetric inhibition of adsorption; on $\mathrm{Pt}(100)$ and $\mathrm{Pt}(110)$, in addition, an adsorbate-induced phase transition turned out to be crucial for the dynamical behavior.

At low pressures ( $\$ 10^{-4}$ mbar) and typical temperatures $(\approx 500 \mathrm{~K})$ there are about $10^{6}$ site changes due to diffusive hopping of an adsorbed $\mathrm{CO}$ molecule per adsorption event, so that the dynamics is reaction- rather than diffusioncontrolled. Therefore the surface can be regarded as being locally well-mixed (i.e., on the order of the diffusion length which amounts to $\lesssim 1 \mu \mathrm{m}){ }^{4}$. Simple mean-field models in the form of reaction-diffusion equations should thus be applicable and have indeed been very successful in reproducing a large number of experimental findings, such as bistability, oscillations, fronts, pulses, spirals, solitary waves, or turbulence, while internal (thermal) fluctuations were not found to play an important role for the description of these phenomena.

However, when looking at very small length scales, and hence at very few adsorption sites, e.g., when studying the

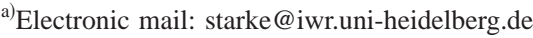

reaction on a field emitter tip, ${ }^{5}$ fluctuations become crucial and stochastic models are required. Moreover, since the adsorption rates grow proportionally to the partial pressures, while surface diffusion remains unchanged, a description with standard reaction-diffusion equations is expected to become less accurate when pressures are increased. The diffusion length may, at higher pressures, decrease until it reaches a scale on which fluctuations become important, so that again stochastic models should be more appropriate.

Our aim in the present study is to develop a stochastic model which, in its deterministic limit, reproduces or even improves the behavior of the well-established reactiondiffusion models for $\mathrm{Pt}(110)$ and $\mathrm{Pt}(100)$, while it remains applicable up to atmospheric pressures as well as at small length scales. To this end, the model should be rescalable, which means the system size and the length scale on which diffusion is an efficient mixing mechanism should enter as parameters. This is achieved by dividing the surface into cells of variable size which are assumed to be well-mixed. The behavior inside each cell as well as the exchange of particles with neighboring ones is stochastic. For large enough cells, fluctuations are averaged out, while their effect can be studied by reducing the number of sites per cell.

Throughout the present paper we consider only the behavior inside one single cell, i.e., the spatially homogenous case. The inclusion of diffusional exchange between cells will be the subject of future work.

In Sec. II the implementation of the model is presented and its deterministic limit derived. In Sec. III we analyze the bifurcation behavior of the deterministic limit, before giving some stochastic simulations in Sec. IV which reveal certain types of noise-induced oscillations not present in deterministic models. Finally, the results are discussed and compared to earlier models. 


\section{MODELING}

In the following we develop a stochastic model for the oxidation of $\mathrm{CO}$ on low-index platinum single crystal surfaces. It was found in UHV experiments that the reaction follows a Langmuir-Hinshelwood mechanism,

$$
\begin{aligned}
& \mathrm{CO}+\star \rightleftarrows \mathrm{CO}_{\mathrm{ad}}, \\
& \mathrm{O}_{2}+2 \star \rightarrow 2 \mathrm{O}_{\mathrm{ad}}, \\
& \mathrm{CO}_{\mathrm{ad}}+\mathrm{O}_{\mathrm{ad}} \rightarrow \mathrm{CO}_{2}+2 \star,
\end{aligned}
$$

where $\star$ is a vacant adsorption site. In addition, the adsorbate-induced phase transition $1 \times 2 \rightleftarrows 1 \times 1$ for $\operatorname{Pt}(110)$ and hex $\rightleftarrows 1 \times 1$ for $\operatorname{Pt}(100)$ has to be taken into account because the surface structure influences the reactivity. We adopt a mesoscopic point of view; instead of distinguishing every single adsorption site, as one would do in microscopic Monte Carlo simulations or in a lattice gas approach, the surface is, in general, divided into several cells of mesoscopic size which are assumed to be well-mixed. (For an application of this approach to the kinetic theory of adsorbates, see Ref. 6.) However, we focus here on one single cell. Changes in the respective numbers of adsorbed $\mathrm{CO}$ molecules, oxygen atoms, and adsorption sites in a nonreconstructed $(1 \times 1)$ surface structure in these cells are described by stochastic birthdeath processes. ${ }^{7,8}$ The deterministic version of the model then results as limit of the stochastic dynamics for large particle numbers.

\section{A. Stochastic model}

The state of a cell containing $N$ adsorption sites at time $t \geqslant 0$ is described by

$$
\mathbf{X}_{N}(t)=\left(\begin{array}{c}
N_{\mathrm{CO}}(t) \\
N_{\mathrm{O}}(t) \\
N_{1 \times 1}(t)
\end{array}\right) \in S_{N}=\{0, \ldots, N\}^{3}
$$

where $N_{\mathrm{CO}}, N_{\mathrm{O}}$, and $N_{1 \times 1}$ denote the numbers of $\mathrm{CO}$ molecules, oxygen atoms, and adsorption sites in a nonreconstructed $(1 \times 1)$ surface structure. In order to characterize a stochastic process corresponding to the reaction mechanisms, transition probabilities to other states $P\left[\mathbf{X}_{N}(t+h)=\mathbf{X}_{N}(t)\right.$ $\left.+\delta \mathbf{X}_{N} \mid \mathbf{X}_{N}(t)\right]$ for small $h$ have to be specified. We introduce the concentrations $u_{N}:=N_{\mathrm{CO}} / N, \quad v_{N}:=N_{\mathrm{O}} / N$, and $w_{N}$ $:=N_{1 \times 1} / N$; in the limit $N \rightarrow \infty$ they are denoted by $u, v$, and $w$.

The terms appearing below in the transition probabilities concerning $N_{\mathrm{CO}}$ and $N_{\mathrm{O}}$ essentially correspond to those used for the (deterministic) reconstruction model for $\operatorname{Pt}(110)$ in Ref. 9. However, the dynamics of the phase transition is modelled in a different way. The parameters and rate constants are defined in Tables II and III. For further discussions see Refs. 9-11.

\section{Adsorption}

- Birth of a $\mathrm{CO}$ molecule on the surface. $N_{\mathrm{CO}} \rightarrow N_{\mathrm{CO}}+1$.

$$
\begin{aligned}
P\left[\mathbf{X}_{N}(t+h)\right. & \left.=\mathbf{X}_{N}(t)+(1,0,0) \mid \mathbf{X}_{N}(t)\right] \\
& =N p_{\mathrm{CO}} \kappa_{\mathrm{CO}} s_{\mathrm{CO}}\left(1-u_{N}^{\xi}(t)\right) h+o(h) .
\end{aligned}
$$

- Birth of two oxygen atoms.

$$
\begin{aligned}
N_{\mathrm{O}} \rightarrow N_{\mathrm{O}}+2 . & \\
P\left[\mathbf{X}_{N}(t+h)=\right. & \left.\mathbf{X}_{N}(t)+(0,2,0) \mid \mathbf{X}_{N}(t)\right] \\
= & N p_{\mathrm{O}_{2}} \frac{1}{2} \kappa_{\mathrm{O}}\left(s_{\mathrm{O}}^{\mathrm{rec}}\left(1-w_{N}(t)\right)+s_{\mathrm{O}}^{1 \times 1} w_{N}(t)\right) \\
& \times\left(\left(1-u_{N}(t)\right)\left(1-v_{N}(t)\right)\right)^{2} h+o(h) .
\end{aligned}
$$

A slight change, as compared to Ref. 9, has been applied to the term describing the adsorption of oxygen. By assuming the adsorption rate of $\mathrm{CO}$ to be proportional to $1-u^{\xi}$ independent of the oxygen coverage as in Ref. 9, one implicitly drops the conservation constraint imposed on $N_{\mathrm{O}}, N_{\mathrm{CO}}$, and the number of vacant sites by the pure LangmuirHinshelwood mechanism. On the other hand, it has been observed experimentally that the presence of oxygen on the surface does not noticeably influence the adsorption of CO. ${ }^{3,12}$ Consequently, we assume that $\mathrm{CO}$ molecules can also be adsorbed at O-covered sites, whereas the dissociative adsorption of an oxygen molecule can only take place at two neighboring free sites. The probability that a particular site is occupied neither by $\mathrm{CO}$ nor by $\mathrm{O}$ is then $(1-u)(1-v)$ instead of $1-u-v$. Since the first term is larger only by $u v$, the difference is negligible if $u$ or $v$ is small. The effect of this alternation of the inhibition factor on the dynamics will be discussed in Sec. III.

The exponent $\xi$ is introduced formally to model a precursor effect in the adsorption of $\mathrm{CO}$ as in Ref. 9, but it was always set equal to 1 in the present computations. Note that the sticking coefficient of oxygen on the $1 \times 1$ phase is higher than on the reconstructed phase which can lead to oscillatory, doubly metastable, and excitable behavior of the reaction kinetics.

\section{Desorption}

- Death of a CO molecule through desorption.

$$
\begin{aligned}
N_{\mathrm{CO}} \rightarrow N_{\mathrm{CO}}-1 . & \\
P\left[\mathbf{X}_{N}(t+h)=\right. & \left.\mathbf{X}_{N}(t)+(-1,0,0) \mid \mathbf{X}_{N}(t)\right] \\
= & N\left(k_{\mathrm{des}}^{\mathrm{rec}}\left(1-w_{N}(t)\right)+k_{\mathrm{des}}^{1 \times 1} w_{N}(t)\right) \\
& \times u_{N}(t) h+o(h) .
\end{aligned}
$$

The desorption of $\mathrm{CO}$ molecules from the reconstructed and the $1 \times 1$ phase has been distinguished here because the difference in the binding energies cannot be neglected in the case of $\operatorname{Pt}(100)$.

\section{Reaction}

- Death of a CO molecule and an oxygen atom through reaction.

$$
\begin{aligned}
& N_{\mathrm{CO}} \rightarrow N_{\mathrm{CO}}-1, N_{\mathrm{O}} \rightarrow N_{\mathrm{O}}-1 . \\
& \begin{aligned}
P\left[\mathbf{X}_{N}(t+h)\right. & \left.=\mathbf{X}_{N}(t)+(-1,-1,0) \mid \mathbf{X}_{N}(t)\right] \\
& =N k_{\mathrm{re}} u_{N}(t) v_{N}(t) h+o(h) .
\end{aligned}
\end{aligned}
$$


(a)

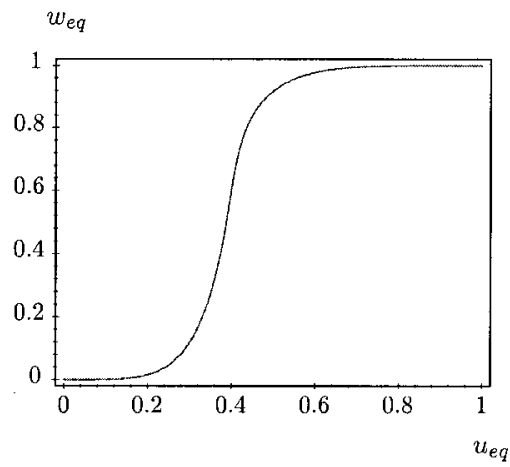

(c)

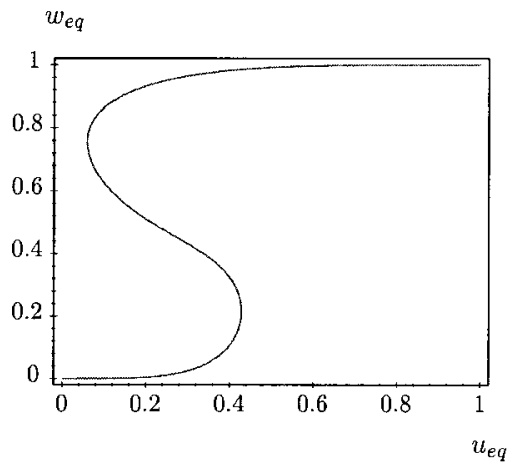

(b)

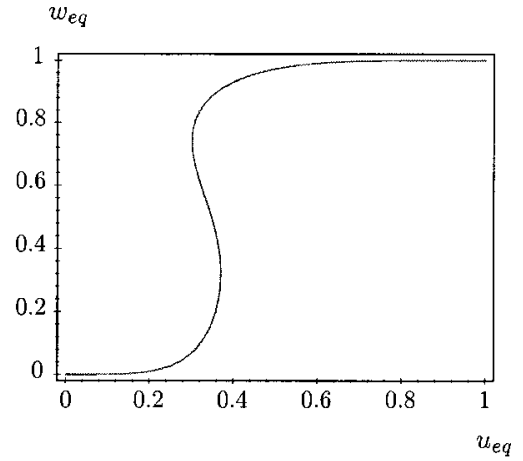

(d)

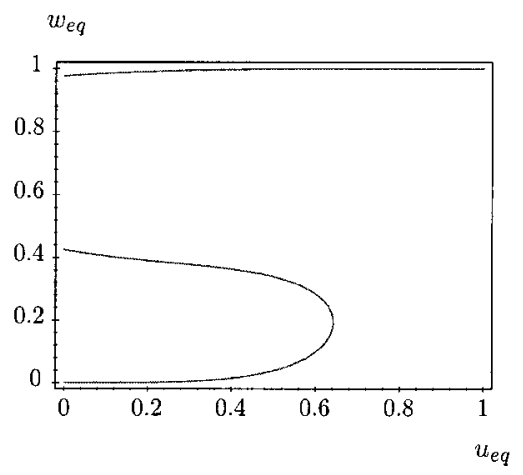

FIG. 1. Equilibria of the kinetics for $v \equiv 0, k_{\mathrm{rec}} / k_{1 \times 1}$ $=0.2, \lambda=4, k_{\mathrm{des}}^{\mathrm{rec}}=k_{\mathrm{des}}^{1 \times 1}$, at different values of $\epsilon$. (a) $\epsilon=0.1$, (b) $\epsilon=0.3$, (c) $\epsilon=0.6$, (d) $\epsilon=0.9$. Introducing different binding energies for $\mathrm{CO}$ on $1 \times 1$ - and the reconstructed phase as in Sec. III B, and thus a coupling between $u$ and $w$, had almost no effect on the curves.
For a well-mixed patch the reaction probability is proportional to the product of the concentrations (mass action kinetics).

\section{Phase transition}

- Death of an adsorption site in a $1 \times 1$ patch and birth of a site in a reconstructed patch.

$$
\begin{aligned}
& N_{1 \times 1} \rightarrow N_{1 \times 1}-1 . \\
& \begin{aligned}
P\left[\mathbf{X}_{N}(t+h)\right. & \left.=\mathbf{X}_{N}(t)+(0,0,-1) \mid \mathbf{X}_{N}(t)\right] \\
& =N k_{\text {rec }} f_{\text {rec }}\left(u_{N}(t), w_{N}(t)\right) w_{N}(t) h+o(h) .
\end{aligned}
\end{aligned}
$$

- Death of a site in a reconstructed patch and birth of a site in a $1 \times 1$ patch.

$N_{1 \times 1} \rightarrow N_{1 \times 1}+1$.

$$
\begin{aligned}
P\left[\mathbf{X}_{N}(t+h)=\right. & \left.\mathbf{X}_{N}(t)+(0,0,1) \mid \mathbf{X}_{N}(t)\right] \\
= & N k_{1 \times 1} f_{1 \times 1}\left(u_{N}(t), v_{N}(t)\right) \\
& \times\left(1-w_{N}(t)\right) h+o(h),
\end{aligned}
$$

where

$$
f_{\text {rec }}\left(u_{N}, w_{N}\right)=(1-\epsilon)\left(1-u_{N}\right)^{\lambda}+\epsilon\left(1-w_{N}\right)^{\lambda},
$$

and

$$
f_{1 \times 1}\left(u_{N}, w_{N}\right)=(1-\epsilon) u_{N}^{\lambda}+\epsilon w_{N}^{\lambda}, \quad \lambda \geqslant 1, \quad \epsilon \in[0,1] .
$$

A major change, as compared to previous work, concerns the treatment of the phase transition. In Ref. 9 it was assumed that, in the absence of oxygen in the gas phase, the equilibrium fraction of nonreconstructed surface can be expressed as a function of the $\mathrm{CO}$ coverage, $w_{\mathrm{eq}}=f\left(u_{\mathrm{eq}}\right)$. In the nonequilibrium case, the dynamics of $w$ was described by $\dot{w}$ $\propto f(u)-w$. However, the ansatz cannot be carried over to $\mathrm{Pt}(100)$ because hysteresis in the phase transition is observed experimentally, and thus $w_{\text {eq }}$ is not expressible as a function of $u_{\text {eq }}$. This can be remedied by assuming $u_{\text {eq }}=g\left(w_{\text {eq }}\right)$ and taking $\dot{w} \propto u-g(w)$ as in Ref. 13, but such an approach is hard to justify in physical terms.

Our model is motivated by the fact that the phase transition proceeds via nucleation and growth. The probability for nucleation is determined solely by the $\mathrm{CO}$ coverage, but the growth of a phase can to some extent be autocatalytic which leads to a dependence of the rate of growth on the concentration of the phase itself. Therefore we choose, e.g., for the growth rate of the $1 \times 1$ phase on a reconstructed surface, a weighted sum of $u$ and $w$ to some power $\lambda$, respectively [cf. Eq. (9)]. A highly nonlinear dependence of this rate on $u$ has been obtained experimentally by Hopkinson et $a l .{ }^{14}$ They measured the growth rate of the $1 \times 1$ phase on a hex- $R$ reconstructed $\mathrm{Pt}(100)$ surface to depend on the $\mathrm{CO}$ coverage on the hex- $R$ phase to a power of about 4.5 . It is plausible that the exponent for $w$ should be of the same order of magnitude. The reverse transition is modeled in an analogous way. In order not to introduce too many parameters, the same $\epsilon$ and $\lambda$ are used, however, the rate constants are allowed to be different.

The effect of the weight $\epsilon$ is shown in Fig. 1. For $\epsilon=0.1$, the equilibrium portion of the $1 \times 1$ phase can be expressed as a function of $u_{\mathrm{eq}}$, as expected. At higher values of $\epsilon$ both phases are stable within a certain range of $u_{\mathrm{eq}}$, for $\epsilon=0.9$ even at arbitrarily small values of $u_{\text {eq }}$.

Transition probabilities to other states behave like $o(h)$. (It is also assumed that the probability for a transition is zero if it would lead out of the state space.) Given an initial distribution for $\mathbf{X}_{N}(0)$, there is a Markov jump process in con- 
tinuous time with state space $S_{N}$ whose transition probabilities satisfy the above equations. Its paths can be chosen right-continuous with left limits. Because the state space $S_{N}$ is bounded, explosions of particle numbers cannot occur, and the process is well-defined for $t \in[0, \infty) .{ }^{15}$ The time evolution of $P\left[\mathbf{X}_{N}(t)=\mathbf{Y} \mid \mathbf{X}_{N}(0)=\mathbf{X}_{0}\right], \mathbf{Y}, \mathbf{X}_{0} \in S_{N}$, is governed by a master equation. ${ }^{7,16}$

\section{B. Deterministic limit}

We denote the stochastic processes describing the dynamics of the densities by

$$
\mathbf{x}_{N}(t)=\frac{1}{N} \mathbf{X}_{N}(t)=\left(\begin{array}{c}
u_{N}(t) \\
v_{N}(t) \\
w_{N}(t)
\end{array}\right) .
$$

Note that all transition intensities [cf. Eqs. (2)-(7)] are proportional to a product of the system size $N$ and a function of the concentrations only. Because of this scaling property it can be proved rigorously that, if $\lim _{N \rightarrow \infty} \mathbf{x}_{N}(0)=\mathbf{x}_{0} \in[0,1]^{3}$ holds with probability one, the paths of the stochastic processes $\mathbf{x}_{N}(t)$ approximate the solution $\mathbf{x}(t)$ of the initial value problem given by the system of ODEs,

$\dot{u}=p_{\mathrm{CO}} \kappa_{\mathrm{CO}} s_{\mathrm{CO}}\left(1-u^{\xi}\right)-\left(k_{\mathrm{des}}^{\mathrm{rec}}(1-w)+k_{\mathrm{des}}^{1 \times 1} w\right) u-k_{\mathrm{re}} u v$,

$\dot{v}=p_{\mathrm{O}_{2}} \kappa_{O}\left(s_{\mathrm{O}}^{\mathrm{rec}}(1-w)+s_{\mathrm{O}}^{1 \times 1} w\right)((1-u)(1-v))^{2}-k_{\mathrm{re}} u v$,

$\dot{w}=k_{1 \times 1} f_{1 \times 1}(u, w)(1-w)-k_{\mathrm{rec}} f_{\mathrm{rec}}(u, w) w$,

and the initial condition $\mathbf{x}(0)=\mathbf{x}_{0}$ at large particle numbers in the following sense:

For arbitrary $t \geqslant 0: \lim _{N \rightarrow \infty} \sup _{0 \leqslant s \leqslant t}\left|\mathbf{x}_{N}(s)-\mathbf{x}(s)\right|$

$=0$, with probability one.

In addition, a central limit theorem can be shown which states that fluctuations of the concentrations vanish like $O(1 / \sqrt{N})$. The proofs follow directly from theorems by Kurtz. ${ }^{17,18}$ A nonrigorous approach to these results is known in the physical literature as van Kampen's system size expansion. ${ }^{7}$

\section{NUMERICAL BIFURCATION ANALYSIS}

In this section we present the results of a numerical bifurcation analysis of system (11) with parameters appropriate for $\operatorname{Pt}(110)$ and $\operatorname{Pt}(100)$. The computations were performed using algorithms contained in the AUTO 97 package by Doedel et al. ${ }^{19}$ Abbreviations of the bifurcations found are listed in Table I, for details see Refs. 20-22. By a degenerate Hopf bifurcation we mean the one described in Ref. 22.

\section{A. $\operatorname{Pt}(110)$}

For $\mathrm{Pt}(110)$, bifurcation diagrams in $p_{\mathrm{CO}}$ and $p_{\mathrm{O}_{2}}$ have been computed for fixed sample temperatures varying from $480 \mathrm{~K}$ to $560 \mathrm{~K}$ in steps of $20 \mathrm{~K}$. The kinetic parameters used are listed in Table II, and quantitative results as well as qualitative sketches are displayed in Figs. 2-4.
TABLE I. Commonly used denotations of bifurcations and their abbreviations.

\begin{tabular}{lcl}
\hline \hline \multicolumn{1}{c}{ Bifurcation } & Codimension & Abbreviation \\
\hline Hopf bifurcation (supercritical) & 1 & $\mathrm{~h}$ \\
Hopf bifurcation (subcritical) & 1 & $\mathrm{~h}^{\prime}$ \\
Saddle-node & 1 & $\mathrm{sn}$ \\
Saddle loop & 1 & $\mathrm{sl}$ \\
Homoclinic bifurcation & & \\
Saddle-node/infinite period & 1 & sniper \\
Saddle-node on a loop & & \\
Saddle-node of periodic orbits & 1 & snp \\
Cusp & 2 & $\mathrm{C}$ \\
Takens-Bogdanov bifurcation & 2 & $\mathrm{~TB}$ \\
Degenerate Hopf bifurcation & 2 & $\mathrm{DH}$ \\
Saddle-node loop & 2 & $\mathrm{SNL}$ \\
Neutral saddle loop & 2 & $\mathrm{NSL}$ \\
Trace 0 saddle loop & & \\
Takens-Bogdanov-cusp & 3 & $\mathrm{TBC}$ \\
\hline \hline
\end{tabular}

At higher temperatures $(500 \mathrm{~K}-560 \mathrm{~K})$ the bifurcation diagram is mainly organized by a cusp and two TakensBogdanov points. In total there are 12 parameter regions with different dynamical behavior (cf. Fig. 2), but only regions $1-5$ are physically relevant because the remaining regions are too small to be detected in typical experiments. In areas 1, 2, and 3 there is only one attractor: a stable node and an asymptotically stable periodic orbit, respectively. The maximal width of the oscillatory region 2 at $540 \mathrm{~K}$ amounts to $\approx 10 \%$ of the value of $p_{\mathrm{CO}}$ at the supercritical Hopf bifurcation. It decreases towards higher as well as lower temperatures. In regions 4 and 5 two attractors coexist; in region 5 there are two stable nodes, while in region 4 the system reaches either a stable node or a small asymptotically stable

TABLE II. Kinetic parameters for $\mathrm{Pt}(110)$.

$\mathrm{CO}$ adsorption

\begin{tabular}{lll}
\hline Impingement rate & $\kappa_{\mathrm{CO}}$ & $3.135 \times 10^{5} \mathrm{mbar}^{-1} \mathrm{~s}^{-1}$ \\
Sticking coefficient & $s_{\mathrm{CO}}$ & 1 \\
Saturation coverage & $u_{\mathrm{s}}$ & 1 \\
Precursor exponent & $\xi$ & 1
\end{tabular}

$\mathrm{O}_{2}$ adsorption

\begin{tabular}{lll}
\hline Impingement rate & $\kappa_{\mathrm{O}}$ & $5.858 \times 10^{5} \mathrm{mbar}^{-1} \mathrm{~s}^{-1}$ \\
Sticking coefficient & $s_{\mathrm{O}}^{1 \times 2}$ & 0.3 \\
& $s_{\mathrm{O}}^{1 \times 1}$ & 0.6 \\
Saturation coverage & $v_{\mathrm{s}}$ & 1
\end{tabular}

Arrhenius rates $\nu \exp \left(-E / k_{\mathrm{B}} T\right)$

CO desorption $\quad k_{\text {des }} \quad \nu_{\text {des }}=5 \times 10^{13} \mathrm{~s}^{-1} \quad E_{\text {des }}=32.3 \mathrm{kcal} / \mathrm{mol}$ Reaction $\quad k_{\mathrm{re}} \quad \nu_{\mathrm{re}}=5 \times 10^{5} \mathrm{~s}^{-1} \quad E_{\mathrm{re}}=8.1 \mathrm{kcal} / \mathrm{mol}$ $\begin{array}{llll}\text { Phase transition } & k_{1 \times 1} \quad \nu_{1 \times 1}=10^{3} \mathrm{~s}^{-1} & \text { or } 0.35 \mathrm{eV} / \mathrm{molecule} \\ E_{1 \times 1}=6.9 \mathrm{kcal} / \mathrm{mol}\end{array}$ $k_{\text {rec }} \quad \nu_{\text {rec }}=0.2 \times 10^{3} \mathrm{~s}^{-1} \quad \begin{array}{ll}\text { or } 0.30 \mathrm{eV} / \mathrm{molecul} \\ E_{\text {rec }}=6.9 \mathrm{kcal} / \mathrm{mol}\end{array}$ or $0.30 \mathrm{eV} / \mathrm{molecule}$

Phase transition

\begin{tabular}{lll}
\hline Exponent & $\lambda$ & 4 \\
Weight & $\epsilon$ & 0.1
\end{tabular}




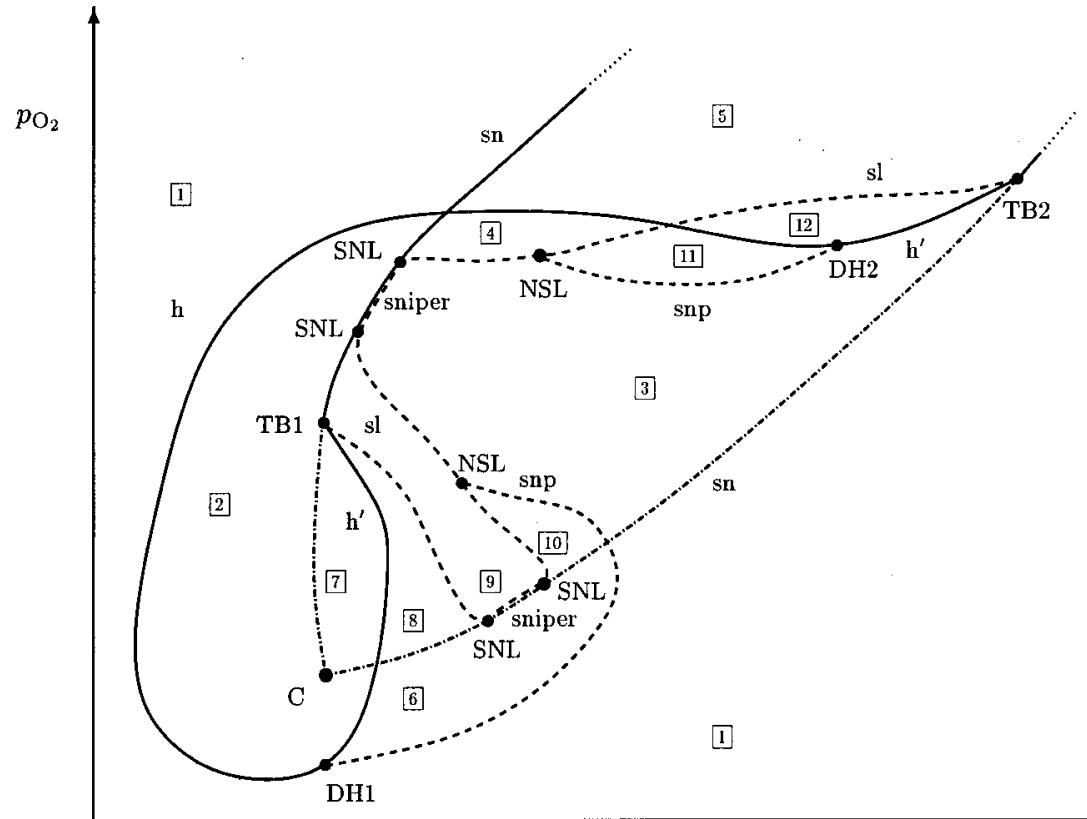

$p_{\mathrm{CO}}$
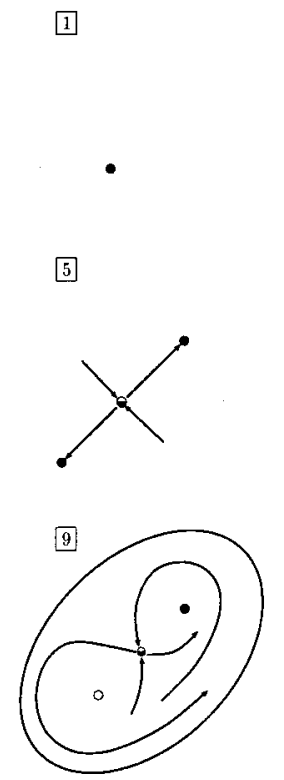

2
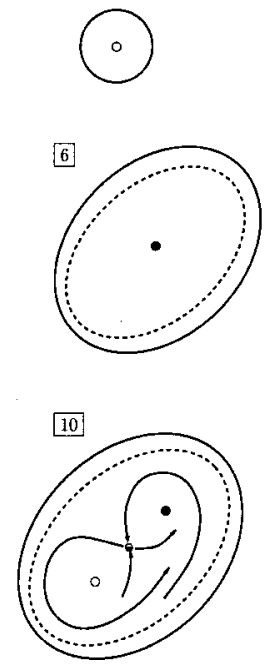

3

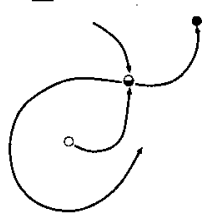

7

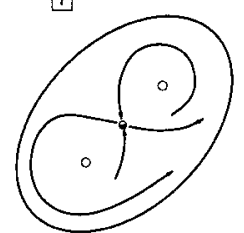

11]

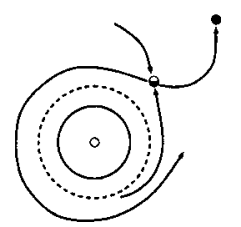

4

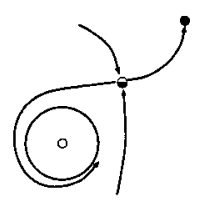

8

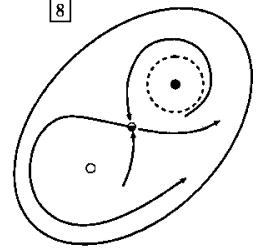

12

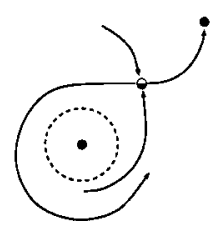

FIG. 2. Sketches of the complete bifurcation diagram at $500-560 \mathrm{~K}$ (top) and phase portraits in different parameter regions (bottom) for $\operatorname{Pt}(110)$. Hopf bifurcations, and saddle-node bifurcations involving a stable node and a saddle with a one-dimensional unstable manifold, are drawn with solid lines, saddle-node bifurcations involving a saddle with a one-dimensional, and another with a two-dimensional unstable manifold are drawn with dash-dotted lines. The dashed curves indicate global bifurcations; cf. Table I. In the phase portraits stable nodes are represented by filled circles, saddle points with a two-dimensional unstable manifold by empty circles, and saddle points with a one-dimensional unstable manifold by half-filled circles. Asymptotically stable periodic orbits are indicated by solid lines, unstable ones by dashed lines. periodic orbit. The width of region 4 is $\$ 1 \%$ of $p_{\mathrm{CO}}$ at 540 $\mathrm{K}$, so it could possibly be detected experimentally. The areas with nontrivial dynamics, such as bistability or oscillations, move towards higher partial pressures as temperature is increased (cf. Fig. 3).

Phase portraits of the dynamics in the different parameter regions are sketched in Fig. 2. In the pictures containing three fixed points the lower one can always be identified with a reactive, only partially nonreconstructed surface with a relatively high oxygen coverage (reactive state), the upper one with a mainly $\mathrm{CO}$-covered $1 \times 1$ surface (poisoned state).

At $480 \mathrm{~K}$ the bifurcation diagram changes also qualitatively. The Takens-Bogdanov point TB1 is now located on the opposite curve of saddle-node bifurcations, and the Hopf bifurcation in its vicinity has changed from sub- to supercritical (cf. Fig. 4) which indicates a Takens-Bogdanov-cusp
(TBC) singularity. Indeed, it seems that the bifurcation diagram at higher temperatures can, at least partly, be understood as a section through a three-dimensional bifurcation diagram corresponding to a TBC. The derivation of such a diagram or tableau is discussed in Ref. 22. The lower part of Fig. 2 (regions 1-10) is contained in the intersection of this three-dimensional tableau with a sphere around the origin in parameter space, whereas regions 11 and 12 are not present there (see also Ref. 23).

Furthermore, the line of Hopf bifurcations is split by two new curves of saddle-node bifurcations, and two additional cusps and Takens-Bogdanov points have appeared. This leads to negligibly small regions with yet different dynamics involving 5 fixed points, while the areas with large periodic orbits have disappeared. Note that the additional sn bifurcations also appear in Ref. 9 at $525 \mathrm{~K}$. 
(a)

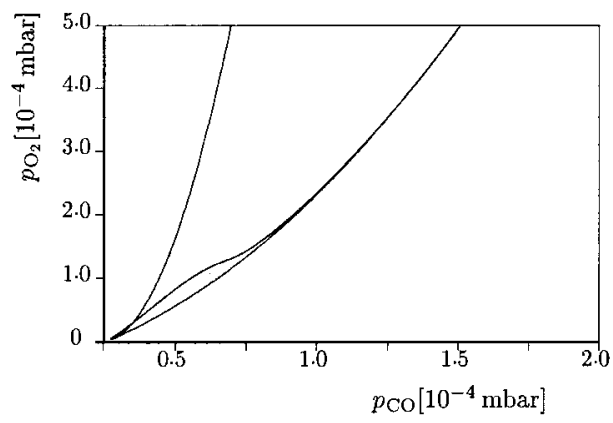

(c)

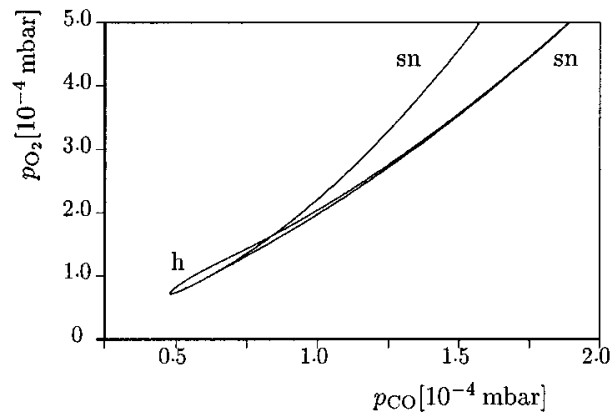

(b)

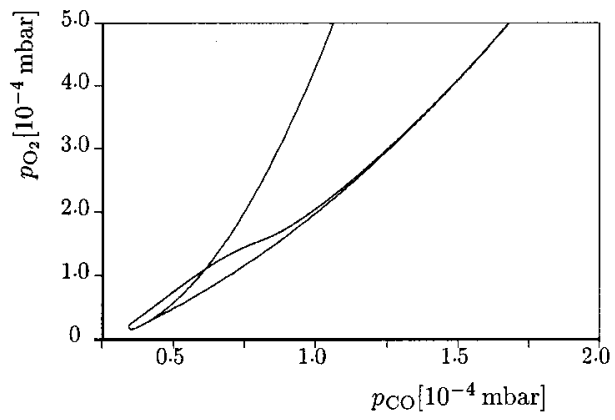

(d)

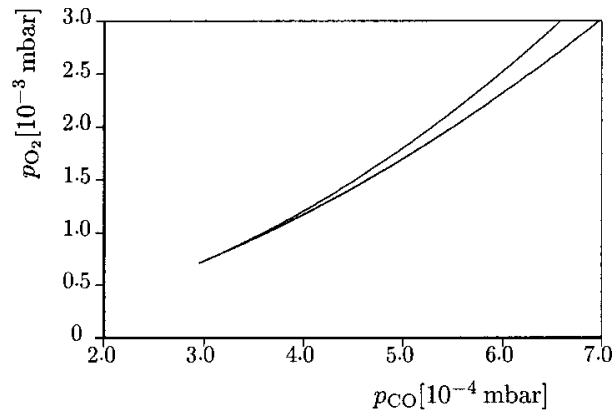

FIG. 3. Quantitative results of the computation of saddle-node and Hopf bifurcations for $\mathrm{Pt}(110)$ from $500 \mathrm{~K}$ (a) to $560 \mathrm{~K}$ (d) in steps of $20 \mathrm{~K}$ (cf. Fig. 2).
From a physical point of view the model presented here yields, apart from quantitative deviations, almost the same results as the one proposed in Ref. 9. In the model analyzed there the point labeled TB1 always lies on the lower curve of saddle-node bifurcations; it approaches the cusp with increasing temperature, but does not cross it. However, this leads only to differences concerning the narrow regions with large periodic orbits. The most important distinction is that here the two curves of saddle-node bifurcations do not merge in a second cusp when $p_{\mathrm{CO}}$ and $p_{\mathrm{O}_{2}}$ are increased, rather bistability persists even at atmospheric pressures. In fact, this is due to the alternation of the adsorption kinetics of oxygen, as was checked by repeating the computation of the saddlenode curves with the term that was used formerly. As in Ref. 9, the dynamics seems to be essentially two-dimensional. A period doubling transition to chaos which was observed experimentally, e.g., could not be found.

\section{B. $\operatorname{Pt}(100)$}

In the case of $\operatorname{Pt}(100)$, the difference in the binding energies of $\mathrm{CO}$ on $1 \times 1$ and hex phase must be taken into account. Moreover, some kinetic parameters have to be changed, most importantly, the sticking coefficient of oxygen on the hex phase is much smaller than on the $1 \times 2$ phase of $\operatorname{Pt}(110)$. The weight $\epsilon$ is increased to $\epsilon=0.3$, in order to model hysteresis in the phase transition; the influence of adsorbed oxygen on the restructuring processes has been neglected.

The kinetic parameters used have been adapted from an older model $;{ }^{11}$ they are listed in Table III. A bifurcation diagram was computed at $480 \mathrm{~K}$ which is reproduced in Fig. 5.

Note that the oscillatory region is much broader than in the case of $\operatorname{Pt}(110)$. It compares favorably with experiments [cf. Figs. 5(d) and 5(e)]. In contrast to earlier models, ${ }^{11,24}$ no (a)

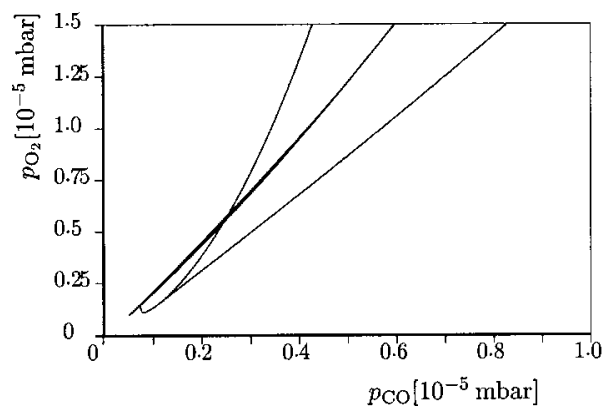

(b)

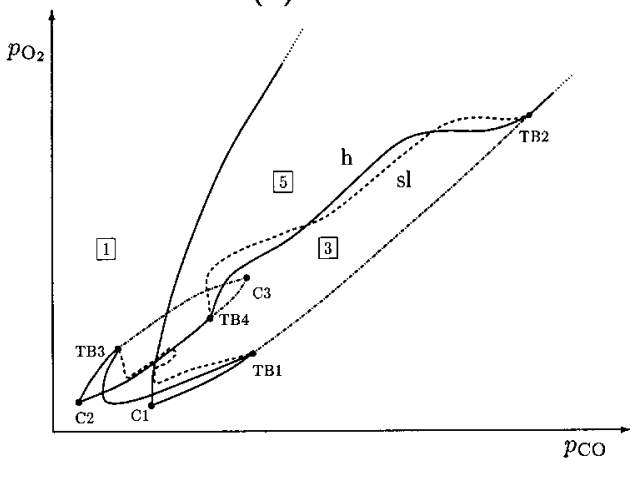

FIG. 4. Bifurcation diagram for $\mathrm{Pt}(110)$ at $480 \mathrm{~K}$, quantitative results (a), and sketch (b) (cf. Fig. 2). The dashed lines indicate the existence of a homoclinic orbit. Curves of snp bifurcations have been omitted. 
TABLE III. Kinetic parameters for $\mathrm{Pt}(100)$ at $480 \mathrm{~K}$.

\begin{tabular}{|c|c|c|}
\hline \multicolumn{3}{|l|}{$\mathrm{CO}$ adsorption } \\
\hline Impingement rate & $\kappa_{\mathrm{CO}}$ & $2.205 \times 10^{5} \mathrm{mbar}^{-1} \mathrm{~s}^{-1}$ \\
\hline Sticking coefficient & $s_{\mathrm{CO}}$ & 1 \\
\hline Saturation coverage & $u_{\mathrm{s}}$ & 1 \\
\hline Precursor exponent & $\xi$ & 1 \\
\hline \multicolumn{3}{|l|}{$\mathrm{O}_{2}$ adsorption } \\
\hline Impingement rate & $\kappa_{\mathrm{O}}$ & $3.75 \times 10^{5} \mathrm{mbar}^{-1} \mathrm{~s}^{-1}$ \\
\hline \multirow[t]{2}{*}{ Sticking coefficient } & $s_{\mathrm{O}}^{\text {hex }}$ & 0.001 \\
\hline & $s_{\mathrm{O}}^{1 \times 1}$ & 0.3 \\
\hline Saturation coverage & $v_{\mathrm{s}}$ & 1 \\
\hline \multicolumn{3}{|l|}{ Arrhenius rates } \\
\hline \multirow[t]{2}{*}{ CO desorption } & $k_{\mathrm{des}}^{1 \times 1}$ & $0.1 \mathrm{~s}^{-1}$ \\
\hline & $k_{\mathrm{des}}^{\text {hex }}$ & $1.0 \mathrm{~s}^{-1}$ \\
\hline Reaction & $k_{\mathrm{re}}$ & $500 \mathrm{~s}^{-1}$ \\
\hline \multirow[t]{2}{*}{ Phase transition } & $k_{1 \times 1}$ & $1.0 \mathrm{~s}^{-1}$ \\
\hline & $k_{\text {rec }}$ & $0.2 \times 1.0 \mathrm{~s}^{-1}$ \\
\hline \multicolumn{3}{|l|}{ Phase transition } \\
\hline Exponent & $\lambda$ & 4 \\
\hline Weight & $\epsilon$ & 0.3 \\
\hline
\end{tabular}

arbitrary parameters have to be introduced through so-called defect terms to get the required behavior. In opposition to $\mathrm{Pt}(110), \operatorname{Pt}(100)$ exhibits two distinct bistable regions. The one at low pressures is due to the hysteresis in the phase transition and extends to zero oxygen pressure, while the one at high pressures is controlled by the reaction dynamics and again does not close. The bifurcation fine structure is somewhat simpler than with $\mathrm{Pt}(110)$, since the lines of Hopf- and sl bifurcations extend from one TB point to the other without the occurrence of any SNL points.

The additional regions with more complicated dynamics are again too narrow to be observed experimentally.

\section{STOCHASTIC SIMULATIONS}

According to the central limit theorem relative fluctuations vanish like $O(1 / \sqrt{N})$, so that the reaction kinetics is well described by the system of ODEs (11) for large enough $N$. Dependent on the values of $p_{\mathrm{CO}}, p_{\mathrm{O}_{2}}$, the sample temperature and the initial condition, either a stable node or a limit cycle is approached. However, simulations for $\mathrm{Pt}(110)$ show that the dynamics of the stochastic model may be qualitatively different to that of the deterministic one for certain choices of parameter values and sufficiently small $N$.

Trajectories of the stochastic processes (10) in continuous time were simulated using an approximating process in discrete time. In order to save computation time, at the expense of accuracy, we did not use one of the standard methods (see, e.g., Ref. 16) which allow only one event per time step. Rather, at each time step $n h \rightarrow(n+1) h$ transitions to all physically accessible states were admitted. These transitions were determined by simulating 6 random variables describing the numbers of adsorption events, desorption events, reactions, and restructuring transitions during the respective time step. The distributions of these random variables were chosen such that the asymptotic behavior of the transition probabilities for $h \rightarrow 0$ proposed in Sec. II A was ensured. The advantage of this method is that the computation time remains essentially independent of the system size $N$. In case of doubt, a simulation can be repeated with smaller $h$; for $h \lesssim 1 / N$ the method is practically the same as the standard method with fixed time step.

Four cases were found in which the stochastic dynamics varies from the deterministic one. In parameter regions where the system is excitable, it does not only fluctuate around a steady state, e.g., in region 1 of Fig. 2, near the supercritical Hopf bifurcation, the process undertakes spontaneous excursions which anticipate the periodic orbit emerging from the stable node [Fig. 6(a)]. Similarly, in the parameter range where the $\mathrm{CO}$-covered surface is excitable excursions can be triggered [Fig. 6(b)]. If the duration of an excursion is long compared to the average time the process fluctuates around the steady state before a new excursion starts, such noise-induced oscillations can appear quite regular. Rectangularly shaped oscillations can be observed in parameter regions with two coexisting attractors. In region 5 the presence of noise leads to transitions between the reactive and the poisoned state [Fig. 6(c)]. In region 4 one of the attractors is a small asymptotically stable periodic orbit which yields mixed-mode oscillations [Fig. 6(d)].

In the case of $\mathrm{Pt}(100)$, simulations revealed that noise can also lead to strong variations in the amplitude of oscillations, making them look irregular.

It must be pointed out that the stochastic model introduced in Sec. II A describes internal (thermal) fluctuations due to the finiteness of the number of adsorption sites. Since for the pressures and temperature used in the simulations the number of sites in a well-mixed cell on surfaces studied in typical experiments should be about $10^{6}$, whereas noiseinduced oscillations could only be obtained for $N \leqq 10^{4}$, we conclude that internal fluctuations on large surfaces are negligible for the parameters chosen, as expected. At low pressures such surfaces are described properly by reactiondiffusion equations and internal fluctuations become significant only in small systems which can be considered as a single cell like, e.g., the $20 \times 200 \AA$ area on a Pt field emitter tip investigated by Suchorski et al., ${ }^{5}$ who indeed observed fluctuation-driven transitions between the reactive and the poisoned state in the bistable parameter range.

At higher pressures, as discussed in the Introduction, the size of the cells which can be regarded as well-mixed on large surfaces has to be chosen smaller; since the model proposed in Sec. II predicts bistability also at high pressures, fluctuation-induced transitions may then occur in single cells. If such transitions happen to occur simultaneously in several neighboring cells, a critical nucleus may form and a front may be triggered.

It is interesting to note that some irregular mixed-mode oscillations of the global coverages which were observed in experiments with a large $\operatorname{Pt}(110)$ surface $^{10}$ at low pressures look quite similar to noise-induced oscillations obtained in the simulations [cf. Figs. 6(d) and 6(e)]. This suggests that some of these oscillations are of stochastic origin. However, mixed-mode oscillations of the global coverages on large 
(a)

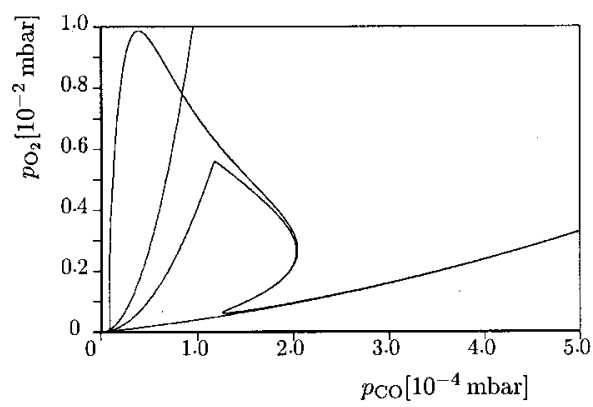

(c)

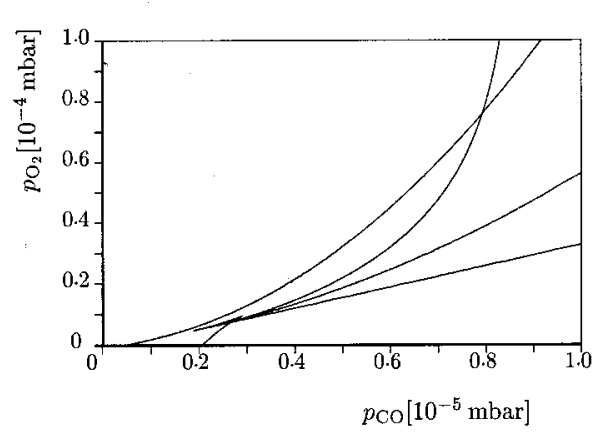

(e)

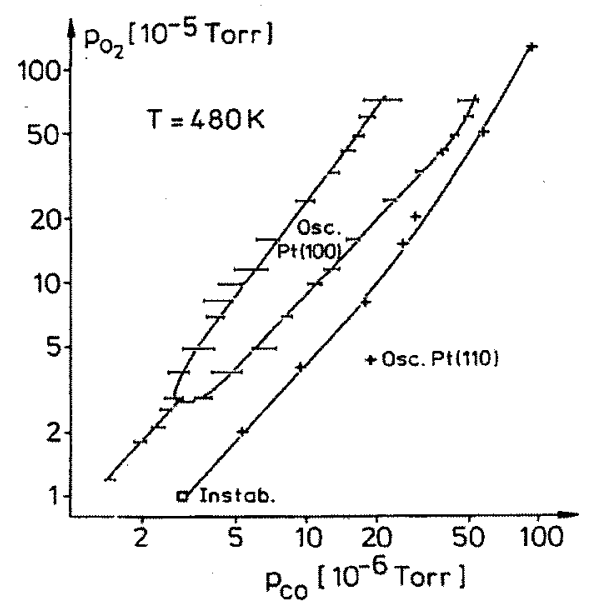

surfaces most likely cannot be traced back to internal noise, and hence the model presented here is not quite appropriate for their description. Nevertheless, if noise of a strength comparable to that used in the simulations is added to external parameters, the whole surface is affected by fluctuations simultaneously, and the effect of such external noise on the dynamics of the global coverages on large surfaces might resemble that of internal noise in small systems described above.

\section{DISCUSSION}

On the preceding pages we presented a stochastic model for the $\mathrm{CO}$ oxidation on low-index platinum single crystal surfaces. A new approach to the phase transition kinetics (b)

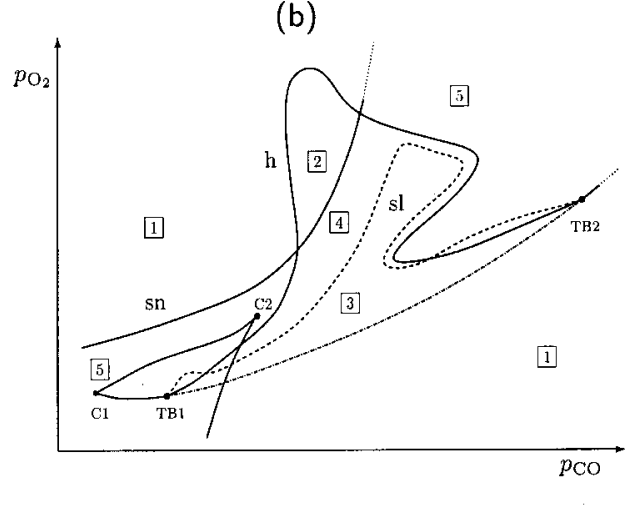

(d)

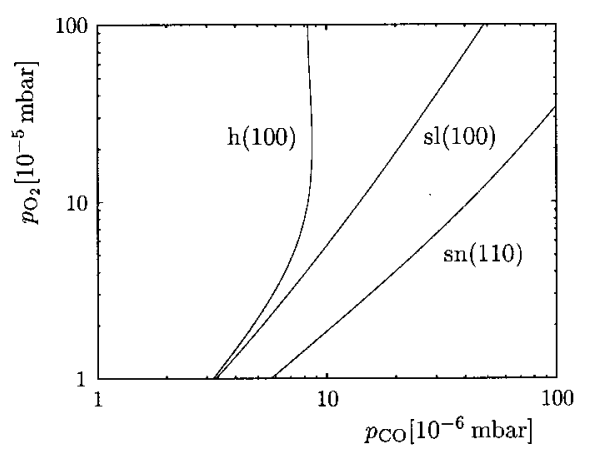

FIG. 5. Bifurcation diagram for $\mathrm{Pt}(100)$ at $480 \mathrm{~K}$. Quantitative results of the computation of sn, Hopf, and sl bifurcations (a), (c), and sketch (b) (cf. Fig. 2). Lines of snp bifurcations have been omitted. In (d) the curves of Hopf and sl bifurcations are plotted using a logarithmic scale. The line on the right is the curve of saddle-node bifurcations corresponding to the loss of stability of the reactive state for $\mathrm{Pt}(110)$. In (e) the corresponding experimental diagram is shown (Ref. 10). allowed a unified description of surfaces of different orientation. A parameter was introduced which weights the respective importance of nucleation and (autocatalytic) growth for the formation of a new phase. For $\operatorname{Pt}(110)$ this parameter was chosen small, which means that nucleation is predominant, whereas for $\mathrm{Pt}(100)$ it was chosen larger which leads to hysteresis effects. A deterministic limit was derived and analyzed for $\mathrm{Pt}(110)$ and $\mathrm{Pt}(100)$. Although no explicit computations were performed, it is expected that the model is also valid for $\operatorname{Pt}(111)$ which does not exhibit an adsorbateinduced phase transition.

For Pt(110), the model yields at low pressures practically the same results as the one proposed in Ref. 9. A slightly different inhibition factor for the adsorption of oxygen was 

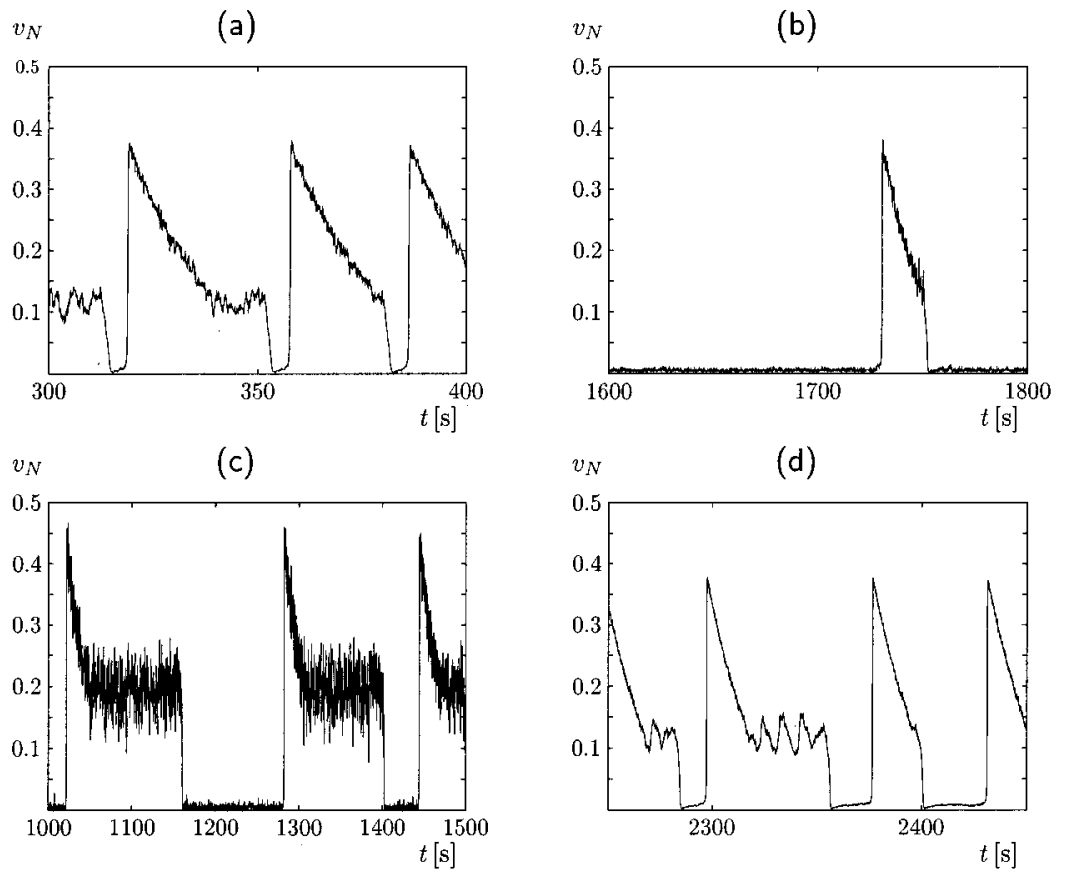

(e)
FIG. 6. Noise-induced oscillations at $520 \mathrm{~K}$ obtained in simulations with $\operatorname{Pt}(110)$ (a)-(d), and mixed-mode oscillations observed in work-function measurements (Ref. 10) at $500 \mathrm{~K}$ (e). (a) $p_{\mathrm{CO}}=3.55 \times 10^{-5} \mathrm{mbar}$, $p_{\mathrm{O}_{2}}=9.6 \times 10^{-5} \mathrm{mbar}, \quad N=10^{4}$; (b) $p_{\mathrm{CO}}=4.3 \times 10^{-5}$ mbar, $p_{\mathrm{O}_{2}}=1.14 \times 10^{-4} \mathrm{mbar}, N=5 \times 10^{3}$; (c) $p_{\mathrm{CO}}=6$ $\times 10^{-5}$ mbar, $p_{\mathrm{O}_{2}}=2 \times 10^{-4} \mathrm{mbar}, N=10^{3}$; (d) $p_{\mathrm{CO}}$ $=4.12 \times 10^{-5} \mathrm{mbar}, \quad p_{\mathrm{O}_{2}}=1.1 \times 10^{-5} \mathrm{mbar}, \quad N=5$ $\times 10^{4}$; (e) $\quad p_{\mathrm{CO}}=6 \times 10^{-6} \mathrm{mbar}, \quad p_{\mathrm{O}_{2}}=1.5$ $\times 10^{-5}$ mbar.

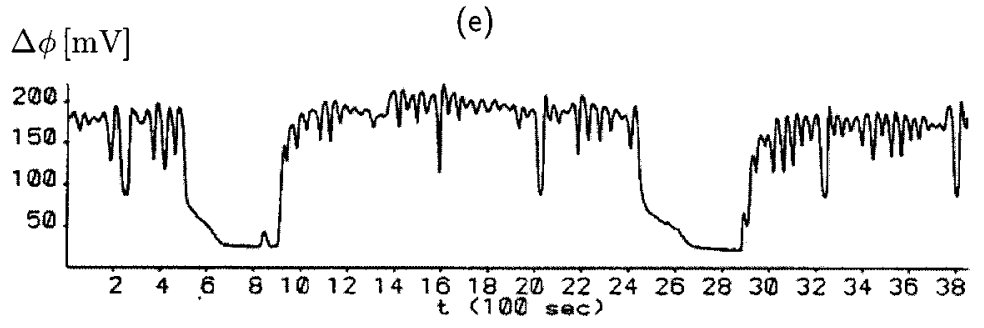

used here which had the effect that bistability persists at higher pressures, in accordance with experimental findings. The ansatz is based on the assumption that $\mathrm{CO}$ and oxygen can, on a short time scale before a reaction takes place, be so close together that they effectively block only one site. For $\mathrm{Pt}(100)$, the measured shape of the oscillatory region is reproduced by the model. Furthermore, it predicts two bistable regions: one at low pressures due to the hysteresis in the phase transition and one at high pressures governed by the reaction kinetics. Both regions have been observed experimentally; the first one most easily at finite $\mathrm{CO}$ and vanishing $\mathrm{O}_{2}$ pressure, excluding the influence of the reaction.

Deterministic mixed-mode oscillations do certainly exist on $\mathrm{Pt}(110)$, as is clear from the observation of the hour-glass effect, ${ }^{25}$ but stochastic simulations (Sec. IV) suggest that some of the rectangularly shaped and mixed-mode oscillations observed in experiments ${ }^{10}$ are of stochastic origin, although the source of the fluctuations is most likely different. The dominant source of noise in the experimental system, as discussed in detail in Ref. 26, presumably stems from surface inhomogeneities; because of steps or impurities small patches of the surface may exhibit slightly different kinetic parameters. In some experiments also pressure fluctuations due to noise in the gas inlet system may have played a role. Nevertheless the effect of such fluctuations is expected to be quite similar to that of the internal fluctuations described here; further investigations based upon stochastic differential equations with noise added to certain parameters would be necessary to clarify this point.
Internal noise during $\mathrm{CO}$ oxidation has recently been observed by Imbihl and co-workers ${ }^{5,27}$ on a Pt field emitter tip in the vicinity of a (110) facet, the observed range thus consisting only of a few hundred sites. The authors observed probability distributions of the coverages quite in agreement with a stochastic model. Note that for the typical time and length scales involved in the pattern formation on single crystal surfaces at low pressures such fluctuations are unimportant. However, with increasing pressures the diffusion length decreases, so that genuinely stochastic behavior can be expected as soon as the pressures are sufficiently high. The inclusion of diffusion, the simulation of spatiotemporal patterns and, in particular, the influence of fluctuations on these phenomena will be the subject of future work.

\section{ACKNOWLEDGMENTS}

One of the authors (J.S.) thanks the Deutsche Forschungsgemeinschaft (SFB 359) for financial support. Financial support by the German-Israeli Foundation (GIF G -622$28.5 / 1999)$ is greatly appreciated. The authors are indebted to J. Christoph and G. Ertl for helpful discussions.

\footnotetext{
${ }^{1}$ R. Imbihl, Heterog. Chem. Rev. 1, 125 (1994).

${ }^{2}$ M. Eiswirth and G. Ertl, "Pattern formation on catalytic surfaces," in Chemical Waves and Patterns, edited by R. Kapral and K. Showalter (Kluwer, Dordrecht, 1995), p. 447.

${ }^{3}$ R. Imbihl and G. Ertl, Chem. Rev. 95, 697 (1995).

${ }^{4}$ M. Eiswirth and H. H. Rotermund, Physica D 84, 40 (1995).

${ }^{5}$ Yu. Suchorski, J. Beben, E. W. James, J. W. Evans, and R. Imbihl, Phys. Rev. Lett. 82, 1907 (1999).
} 
${ }^{6}$ M. Hildebrand and A. S. Mikhailov, J. Phys. Chem. 100, 19089 (1996).

${ }^{7}$ C. W. Gardiner, Handbook of Stochastic Methods (Springer, Berlin, 1985).

${ }^{8}$ H. Haken, Synergetics: An Introduction (Springer, Berlin, 1983).

${ }^{9}$ K. Krischer, M. Eiswirth, and G. Ertl, J. Chem. Phys. 96, 9161 (1992).

${ }^{10}$ M. Eiswirth, "Phänomene der Selbstorganisation bei der Oxidation von CO an Pt(110)," dissertation, Ludwig-Maximilians-Universität München, 1987.

${ }^{11}$ R. Imbihl, M. P. Cox, G. Ertl, H. Müller, and W. Brenig, J. Chem. Phys. 83, 1578 (1985).

${ }^{12}$ G. Ertl, Ber. Bunsenges. Phys. Chem. 98, 1413 (1994).

${ }^{13}$ S. Krömker, "Model and analysis of heterogeneous catalysis with phase transition," dissertation, Universität Heidelberg, 1997.

${ }^{14}$ A. Hopkinson, J. M. Bradley, X.-C. Guo, and D. A. King, Phys. Rev. Lett. 71, 1597 (1993)

${ }^{15}$ L. Breiman, Probability (Addison-Wesley, New York, 1968).

${ }^{16}$ J. Honerkamp, Stochastic Dynamical Systems (VCH, Weinheim, New York, 1994).

${ }^{17}$ T. G. Kurtz, Approximation of Population Processes, Society for Industrial and Applied Mathematics, CBMS-NSF, Regional Conference Series in Applied Mathematics 36, SIAM, 1981.
${ }^{18}$ S. N. Ethier and T. G. Kurtz, Markov Processes: Characterization and Convergence (Wiley, New York, 1986), Chap. 11.

${ }^{19}$ E. Doedel, A. R. Champneys, T. F. Fairgrieve, Y. A. Kusnetsov, B. Sandstede, and X. Wang, AUTO 97: Continuation and bifurcation software for ordinary differential equations, http://ftp.cs.concordia.ca/pub/doedel/auto

${ }^{20} \mathrm{~J}$. Hale and H. Koçak, Dynamics and Bifurcations (Springer, Berlin, 1991).

${ }^{21}$ S. Wiggins, Introduction to Applied Nonlinear Dynamical Systems and Chaos (Springer, Berlin, 1990).

${ }^{22}$ J. Guckenheimer, Physica D 20, 1 (1986).

${ }^{23}$ K. Krischer, "Nichtlineare Dynamik zweier Grenzflächenreaktionen," dissertation, Freie Universität Berlin, 1990.

${ }^{24}$ R. F. S. Andrade, G. Dewel, and P. Borckmans, J. Chem. Phys. 91, 2675 (1989).

${ }^{25}$ M. Eiswirth and G. Ertl, Surf. Sci. 90, 177 (1986).

${ }^{26}$ Th.-M. Kruel, M. Eiswirth, and F. W. Schneider, Physica D 63, 117 (1993).

${ }^{27}$ Yu. Suchorski, J. Beben, R. Imbihl, E. W. James, D.-J. Liu, and J. W. Evans, Phys. Rev. B 63, 165417 (2001). 